\title{
A Review on Wireless Sensor Network Protocol for Disaster Management
}

\author{
M. Sheik Dawood \\ Sethu Institute of \\ Technology \\ Pulloor, India
}

\author{
J. Suganya \\ Sethu institute of \\ Technology \\ Pulloor, India
}

\author{
R. Karthika Devi \\ Sethu institute of \\ Technology \\ Pulloor, India
}

\author{
G. Athisha \\ PSNA College of Engg. \\ \& Technology \\ Dindigul, India
}

\begin{abstract}
Disasters management and emergency services used to protect a person or society from the cost of disasters such as tsunami warning, landslide monitoring, earthquake rescue operation, volcano monitoring, and fire protection. Timely report and responses are especially important for reducing the number of sufferers and damages from incidents. In such cases, the communication structure that may not function well. This makes it hard to gain information about the incident, and then to respond to the incident rapidly and properly. Sensor networks can provide a good solution to these problems through actively monitoring and well-timed reporting emergency incidents to base station. Our objective on this topic aim to study different sensor network protocols to resolve some key technical problems in this area, thus identify the energy efficient wireless sensor network architecture for significant improvement of disaster management. We also analyze the WSN protocol based on metrics such as Energy efficiency, location awareness, network lifetime. It furthermore focuses the advantages and performance for disaster management.
\end{abstract}

Keywords: —Disaster management, Sensor network, Energy Efficiency, Lifetime

\section{INTRODUCTION}

Disaster Management is a colossal task. They could hardly enclose to any particular location that neither do they disappear as quickly they appear. It is important about proper management to optimize efficiency of planning and response. Due to limited resources collective efforts occurred. The level of association requires a coordinated and organized effort to militate against, prepare for, respond to, and recover from emergencies and their effects in the shortest possible time [18].

A disaster is an event of natural or man-made causes that lead to sudden disruption of normalcy within society, causing damage to life and property, to reduce this damage effective management of information is important in the disaster management sector. The sectors from emergency response planning to short-range early warning to long-range mitigation and prevention planning are applied [19].

\section{SENSOR NETWORK FOR LANDSLIDE MONITORING}

Alberto Rosi et al. Proposing their research in "Landslide Monitoring with sensor network". This paper report on the implementation and deployment of a system for Landslide Monitoring in the Northern Italy Apennines and analyze the positive results and achieved it. Here efficient 'data collection algorithm' is used to receive the data correctly when disaster is occurring. [14]

Distributed detection strategy for landslide prediction using WSN". Propose by Prakshep Mehta (2007) et al. The researchers furthermore explained the use of various distributed algorithms for landslide prediction using WSN. The distributed vector based detection with independent cluster (DVBD-IC) algorithm stated that each $\mathrm{CH}$ sends the calculated likelihood ratio ( LR) to the base station through multihop. They assumed that the data from the nodes within the cluster correlated but the data from different clusters are independent. The result of this paper shows that high Energy consumption of WSN protocol. [12].

Rehana Raj $\mathrm{T}$ et al, described their current research in Fault Tolerant energy saving Clustering scheme in WSN for Landslide Area Monitoring to reduce communication and processing overhead. The proposed approach, which organizes the whole network into smaller cluster and sub cluster groups as shown in figure -1 for enabling a considerable reduction of Communication and processing overhead. Sub clusters formation also gives the possibility to deal skillfully with sensor nodes, node leader, and cluster head failures.

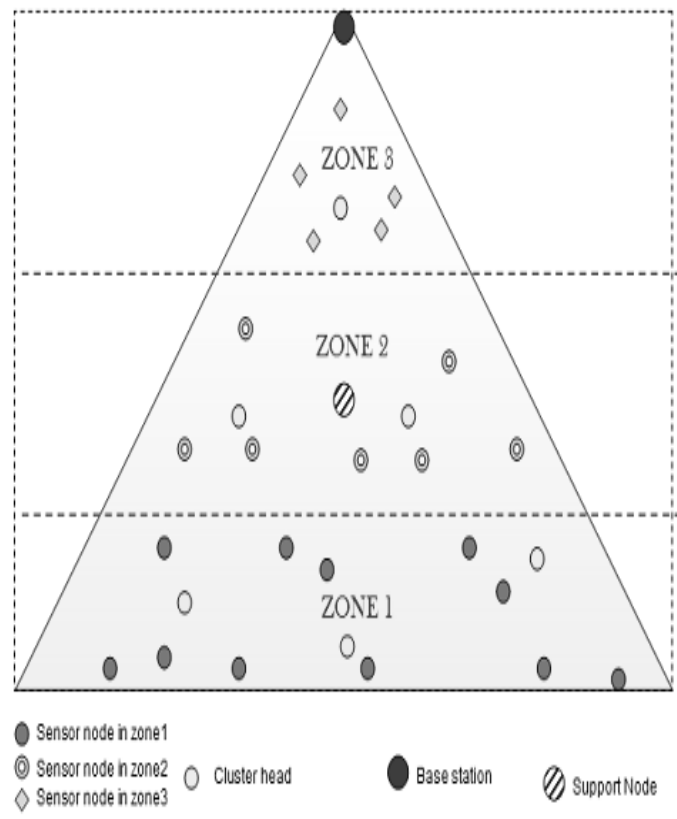

Figure- 1 
On this approach failed data prediction is being achieved by a fuzzy control system [6]. Dominating Set based Algorithm used for fixing Cluster heads to improve the energy efficiency of network.

Siti Khairunniza-Bejo (2011) et al, proposed that "Historical Analysis of the Land Movement in Landslide Area Using Elastic Image Registration and Conditional Statement Approach". An elastic image registration and changeunchanged conditional statements procedure appropriate for historical analysis of the land movement in a landslide area presented herein. Landslide areas detected using the number of pixel movements during the registration process. It shows that the size of pixel movement used to detect changes in landslide areas. The more sequences of changed images were used, and the more information about the history of the area can be gathered [15].

G. P. Ganapathy (2010) et al reported their current research in "Need and Urgency of Landslide Risk Planning for Nilgiri District, Tamil Nadu State, India". The landslide is one of the major natural hazards that are commonly experienced in hilly terrains all over the world. In this, paper Landslide Vulnerability Index Risk analysis involves assessing the threat and these affected the people and property. It furthermore, provided an overview of risk management processes on Landslide Monitoring and relief operation. [16].

Kohei Arai (2012) analyzed their current research in "Sensor Network for Landslide Monitoring with Laser Ranging System, Avoiding Rainfall Influence on Laser Ranging by Means of Time Diversity and Satellite Imagery Data Based Landslide disaster relief". Sensor networks for Landslide Monitoring with a laser ranging system developed together with landslide disaster relief with remote sensing satellite imagery data. Experimental results show that the proposed protocol does work for the situation like rainfall influence and for landslide disaster relief [17].

Energy efficient sensor network protocol for landslide area monitoring proposed by sheik Dawood et.al (2012). The work furthers explained the Energy efficient modulation with two tier clustering Architecture for the fault tolerant sensor network. The improved lifetime of this protocol can be useful for the disastrous condition like Landslide Monitoring and management. [20].

\section{WSN FOR AIR POLLUTION MONITORING}

Kavi k.Khedo (2010) et al. Report on a "WSN air pollution monitoring system (WAPMS)".Indeed with the increasing number of vehicles on our roads and rapid urbanization air pollution has considerably increased in last decades. To reduce this problem they use 'Recursive Converging algorithm' and duplicate elimination technique.both the techniques are represented by authors shown in figure- 2

The algorithm is used to merge data to eliminate duplicates, filter out invalid readings and summarize to simple form which significantly reduce the quantity of data to be transmitted to the sink and thus saving Energy [1].

\section{WSN FOR EMERGENCY RESPONSE}

A Location aware WSN protocol for emergency response task when disaster happens is described by Ashok Kumar (2009). In this paper localization, Communication is the main aspects of disaster-aided network (DAN).In localization aspects, a 'ranging and position estimation methodology' for patient localization at disaster site is proposed. As the result shows that DAN system supports efficient resource planning, quick evacuating of the patient and increase of situation awareness during disaster management [13]

G.Ragunath (2012) et al. Analyze "Deployment of the WSN for real time monitoring and disaster management". It is very essential to have a robot during disaster conditions like an earthquake or Bomb blast, where we have to identify living human beings as quickly as possible to save life; the authors explained the use of two mobile Robots for Surveillance (Target robot) and Rescue operation. The Target robot could easily navigate through rough terrain without being stuck and it can detect obstacles, fire, poisonous gases, Enemy remote vehicles. The Rescuer robot uses to detect a human, using the IR radiation emerging from the live humans and contactless sensor microwave Impulse radar (MIR) for detecting the heartbeat of human. The robot has a scissor Lift for Lifting heavier debris or Metal Rods and rescues the human. This paper is considered as very effective and life saving protocol for earthquake, landslides and other natural disasters [2]

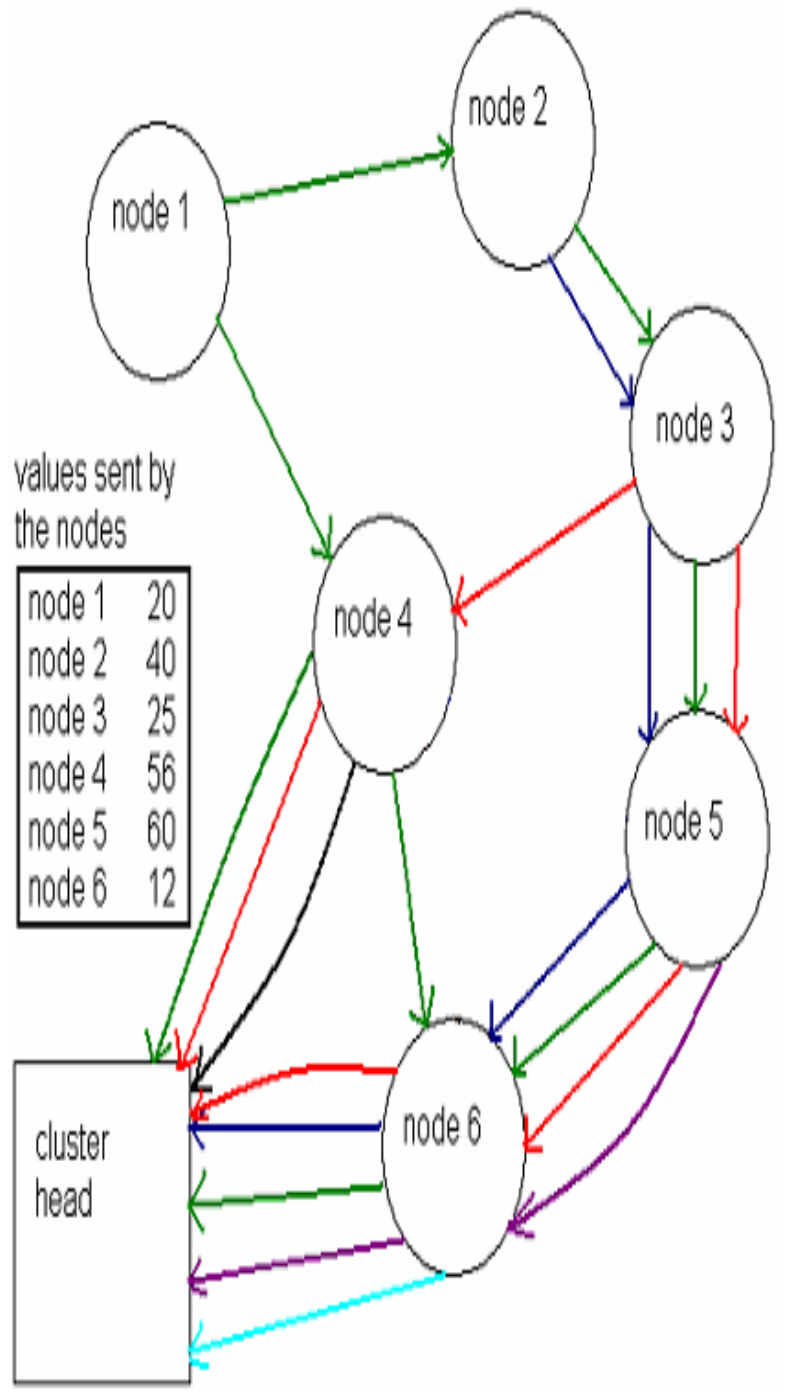


Multihop routing of data during a collection instance and illustration of duplicate elimination technique

\begin{tabular}{|ll|l|}
\hline Node ID & Colour & Value \\
\hline 1 & Green & 20 \\
\hline 3 & Ral & 25 \\
\hline 4 & Blac: & 50 \\
\hline 3 & Red & 25 \\
\hline 2 & Blue & 40 \\
\hline 1 & Green & 20 \\
\hline 5 & Purple & 60 \\
\hline 6 & Cyar. & 12 \\
\hline
\end{tabular}

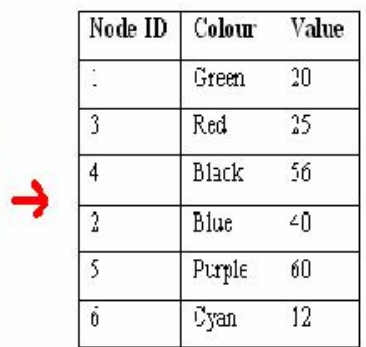

state of table after eiminaticn of duplicates
Initial readirgs collested

\section{duplicates from noce 1}

\section{duplicates from noce 3}

Figure -2

\section{WSN FOR EARTHQUAKE DETECTION}

Rui tan (2010) et al. Describing in their current research in "Quality driven Volcanic Earthquake Detection using WSN". In this paper, they described novel qualities driven approach to achieve real time, long-lived volcanic detection. These approaches based on 'collaborative signal processing algorithm'. The result of this is minimizing sensor's Energy consumption subject to sense quality requirement [11].

Makoto Suzuki (2007) et al. Proposing "A high density earthquake monitoring system using WSNs". For high precision monitoring, they developed Pavenet OS, which is hard real time operating system for sensor nodes, and accelerate the sensor board. In this model work in wireless mode and acceleration sensor board for necessary earthquake monitoring. As a result, they have easily preliminary evaluation of high precision and high density earthquake monitoring system [10].

Naveed Ahmad (2011) et al, propsed a architecture for Adhoc wirelesses Sensor Network for Disaster Survivor Detection as shown in figure-3. Wireless Ad hoc sensor nodes are playing a vital role in wireless data transmission infrastructure.

The proposed model for the disaster survivor detection based on extremely critical disaster situations where this Energy efficient Architecture can successfully trace and locate thousands of people in critical circumstances. The emphasis of this paper focuses on earthquake based disasters. [7].

\section{WSN FOR FORECASTING}

Victor seal (2012) et al. Describing "A simple flood forecasting scheme using wireless sensor network" This work presents a forecasting model designed using WSNs to predict flood in rivers using a simple and fast calculations to provide real time results and, save the lives of people who may be affected by the flood. The novel algorithm is used to predict from the flood forecasting and use the independent number of guidelines. Figure-4 depicts the WSN deployment scheme in a flood prone river basin. The result is to give awareness to the people and, save their life [5].

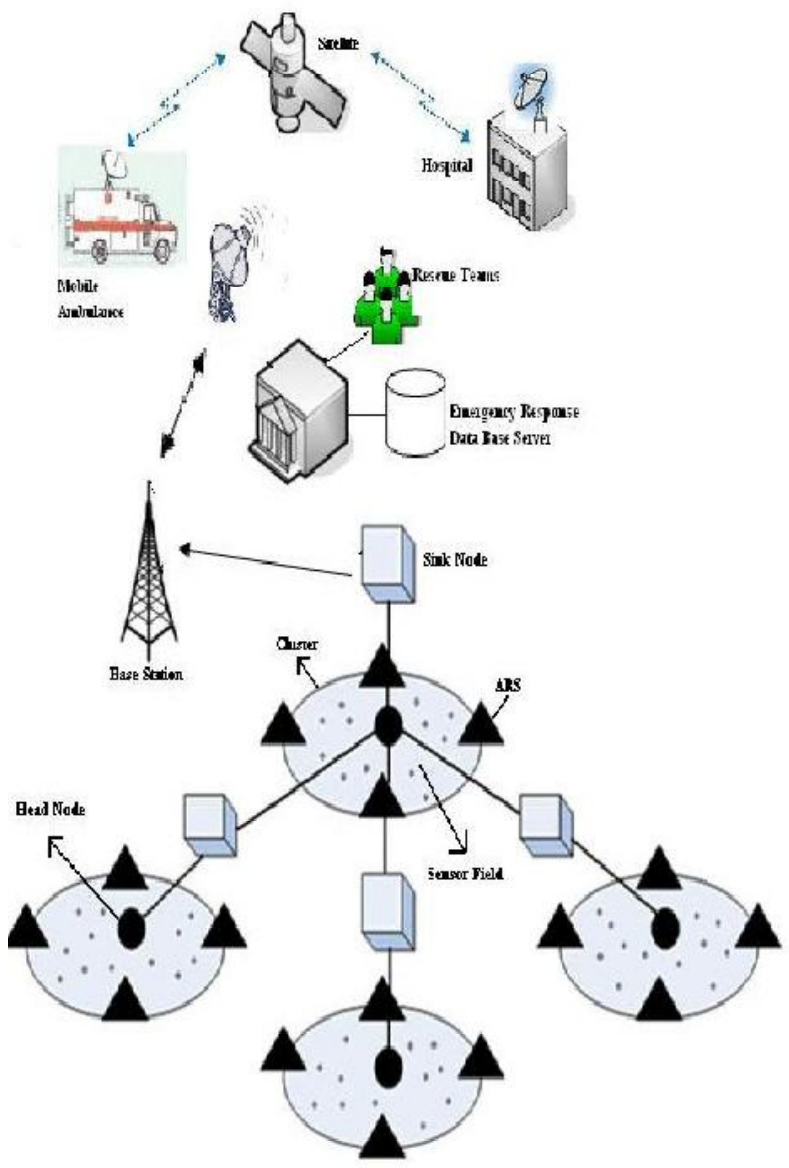

Figure-3

Cholatip Yawut (2011) et al proposed that "A WSN for Weather and disaster Alarm Systems" In this paper, presented a system used to avoid massive damage from natural disasters. In this system, a WSN based on ZIGBEE used as a weather station network sending information and disasters' alerts. This proposed system takes advantage of WSNs that can send signals over far distances by using a mesh topology; this transfer the data and consumes low power. Therefore, this system installed in locations has no access to electricity [4]. 


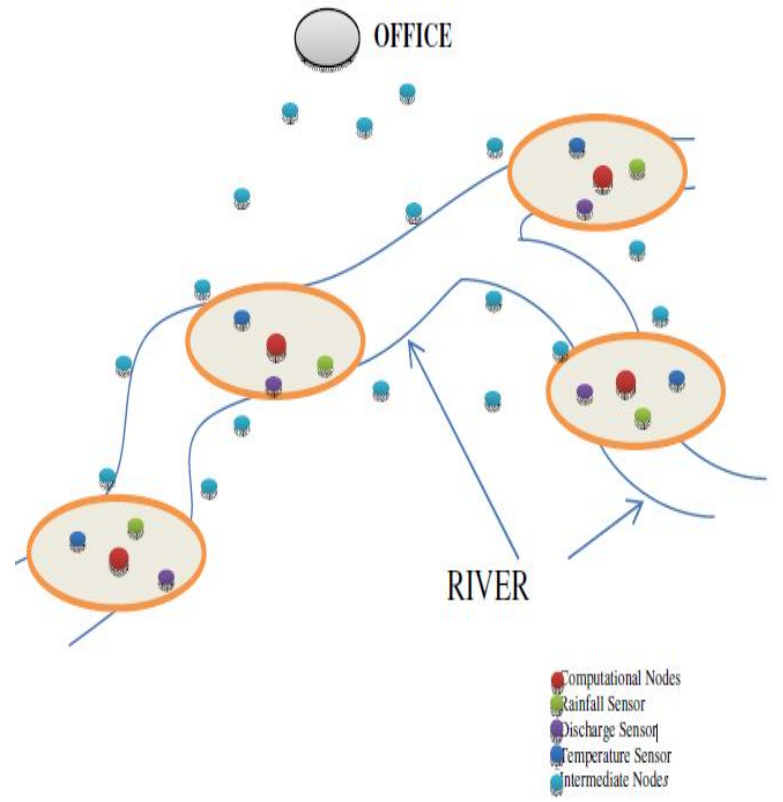

Figure-4

\section{PERFORMANCE ANALYSIS OF DISASTER MANAGEMENT PROTOCOLS}

Mohamed youis (2004) et al, analyzed that "On Handling QOS Traffic in WSN" Many new routing and MAC layer protocols have been proposed for WSNs tackling the issues raised by the resource constrained unattended sensor nodes in large-scale deployments. Transmission of data in such cases requires the Energy and QOS aware network management to ensure efficient usage of the sensor resources and effective access to the gathered measurements. They highlight the architectural and operational challenges of handling of QOS traffic in sensor networks [8].

Sanjay Patel (2011) report On an "Interfacing of Sensor Network to Communication Network for Disaster Management".This work with the sensor network and Communication network for disaster management using GSM modem which the concerned authorities dealing with disaster management get the message on their mobile phones about disaster information. Figure-5 given a general block diagram for Interfacing of Sensor Network to Communication Network for Disaster Management.

The GSM modem sends and receives data through radio waves. Now a day's number of small disasters like fire, chemical leakage, pollution etc, happens frequently and need immediate relief action. The result of this paper release the immediate information for quick action to such events [3].

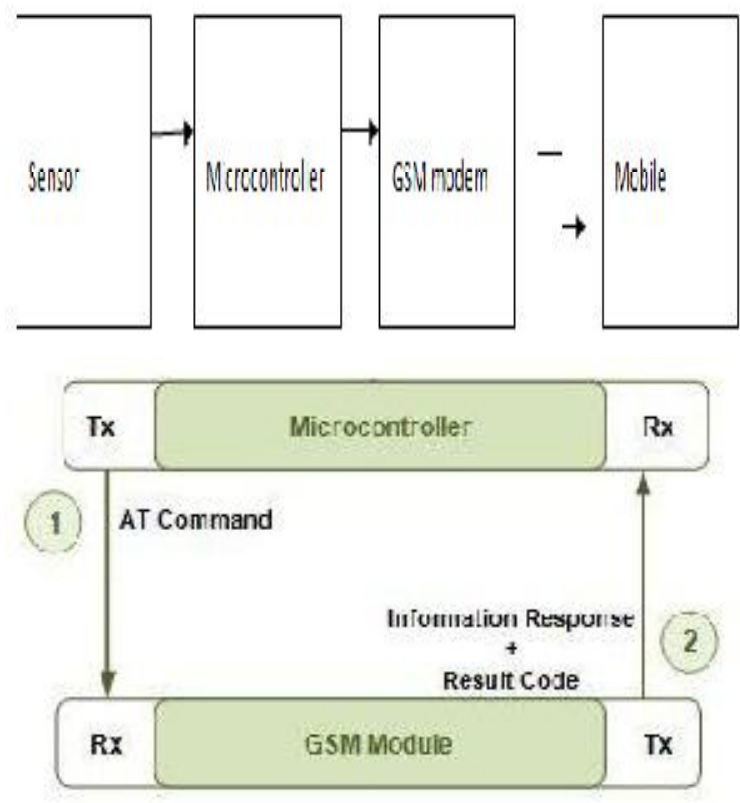

Figure-5

WSNDM is a wireless sensor network protocol for disaster management is proposed by Saha, S. Matsumoto, M. The authors have proposed this protocol with an updated hybrid network framework. In this paper, the performance of WSNDM protocol compared with LEACH.it furthermore gave a solution to collapsed base station problem. In sensor networks, average Energy dissipation measured performance, system lifetime, successful data delivery and the number of live nodes. Their simulation, considered those performance factors. Simulation results prove that WSNDM outperforms LEACH protocol . [22]

A natural disaster management system based on location aware distributed sensor networks is presented by Kumar (2005) to achieve maximum lifetime in WSNs in disaster management applications. The authors propose a system based on hierarchical transmission of packets from sensor nodes to the base station by identifying a path from one head to a subsequent head along the route. The algorithm divides the entire sensor network into logical concentric zones based on Energy of transmission of the packet is transmitted from a head node to one of the head nodes in the next zone with lesser distance. The implementation profoundly uses the location awareness of sensor nodes for better routing and, hence is applicable only to those situations where such data can be made available at the time of installation. They further provide the concept of multiple memberships of sensor nodes to different heads within its area of reach thereby handling disaster conditions where a head fails without notification to its primary members [21]. 


\section{WSN FOR ENVIRONMENTAL MONITORING}

Al-Sakib Khan Pathan (2006) et al. Analyzing "Smartening the Environment is using WSNs in a developing country". In this paper, they explore the prospects of wireless sensor networks and propose a design level framework for developing the smart environment using WSNs. Here update the information about flood, water level, traffic and controlling, environmental frequently. If any changes mean, they used two phases. First phase used to collect the data and send to the local base station. The second phase involves data distribution network, where the processed data sent to different factors involved in network [9].

\section{CONCLUSION}

This survey studies the role of sensor network in disaster management. It furthermore studied the different types of disaster management protocols and their application in extremely disastrous conditions. The performance such protocols are studied based on Energy efficiency, location awareness and network lifetime.

\section{REFERENCES}

[1] Kavi k khedo, Rajiv perseedoss.2010. Avinash Mungar, "A wireless sensor network air pollution monitoring system", International Journal of wireless \&Mobile Networks(IJWMN),Vol.2,No.2.

[2] G. Ragunath \& Dinesh Kumar.2012. "Deployment of the WSN for real time monitoring and disaster management", Indian Journal of Innovation \& developments, Vol.1.

[3] Sanjay Patel, O.P, Vyas.2012. "Interfacing of Sensor Network to Communication Network for Disaster Management" International Journal of Soft computing \& Engineering (IJSCE), Vol.2.

[4] Cholatip yawut \& Sathapath kilaso.2011. "A Wireless Sensor Network for Weather and disaster Alarm Systems" in proceedings of International conference on Information \& Electronics Engineering.

[5] Victor Seal, Arnab Raha, Shovan Maity.2012. "A simple flood forecasting scheme using wireless sensor network", International Journal of Adhoc Sensor \& Ubiquitous Computing (IJASUC) Vol.3, No.1.

[6] Rehna Raj, Maneesha Ramesh, V. and Sangeeth Kumar.2008. "Fault Tolerant Clustering Approaches in Wireless Sensor Network for Landslide Area Monitoring"'in Proceedings of the International Conference on Wireless Networks (ICWN'08), Vol. 1, pp. 107-113

[17] Naveed Ahmad, Naveed Riaz, Mureed Hussain.2011. "Ad hoc wirelesses Sensor Network Architecture for Disaster Survivor Detection", International journal of advance science and technology, vol 34, September 2011.

[8] Mohamed youis.2004."On Handling QoS Traffic in Wireless Sensor Network", in proceedings of the International conference, January 2004.
[9] Al-Sakib Khan Pathan, Choong Seon Hong, Hyung-Woo Lee.2006. "Smartening the Environment using wireless sensor networks in a developing country", IEEE ICACT, Volume I.

[10] Makoto Suzuki, Narito Kurata, Hiroyuki Morikawa, Shunsuke Saruwatari.2007. "A high density earthquake monitoring system using wireless sensor networks", International conference on Embedded network sensor system

[11] Rui Tan, Guoliang Xing, Jinzhu Chen, Wen-Zhan Song, Renjie Huang. 2010. in inproceedings on "Quality driven Volcanic Earthquake Detection using wireless sensor network", Real time system symposium.

[12] Prakshep Mehta, Bhushan Jagyasi, Kalyana Tejaswi, Rajat Bansal, Chandresh Parekh, Anmol Sheth, S. N. Merchant, T. 2007.'Distributed detection strategies for landslide prediction using Wireless sensor networks, in proceedings of first international global information infrastructure Symposium, Morocco. Pp 195-198

[13]Ashok Kumar.2009. "A Location aware wireless sensor network for assisting emergency response to disaster" International journal of computer science and information security, Vol 2, June 2009.

[14] Alberto Rosi, Nicola Bicocchi, Gabriella Castelli, Marco Mamei, Franco Zambonelli..2011. "Landslide Monitoring with sensor network" published in the International journal of signal and imaging systems engineering. Vol. 10 No.3.

[15] Siti Khairunniza-Bejo, Abdul Rashid Mohamed Shariff.2011. "Historical Analysis of the Land Movement in Landslide Area Using Elastic Image Registration and Conditional Statement Approach" International Journal of Multimedia and Ubiquitous Engineering Vol. 6, No. 3.

[16] G. P. Ganapathy, K. Mahendran, S.K Sekar.2010. "Need and Urgency of Landslide Risk Planning for Nilgiri District, Tamil Nadu State, India" International journal of genetics and Geosciences volume 1, no 1 .

[17] Kohei Arai.2012."Sensor Network for Landslide Monitoring With Laser Ranging System Avoiding Rainfall Influence on Laser Ranging by Means of Time Diversity and Satellite Imagery Data Based Landslide Disaster Relief" International Journal of Applied Sciences (IJAS), Vol 3,No.1 .

[18] "Disaster management".2008 an article published online in the Virtual University for Small States of the Commonwealth (VUSSC) .

[19] Aloysius J. Rego.2001. "National Disaster Management Information Systems \& Networks" June 2001.

[20] M. Sheik Dawood, Sajin Salim, S. Sadasivam, G. Athisha.2012."Energy Efficient Modulation Techniques for Fault Tolerant Two-Tiered Wireless Sensor Networks,Journal of Asian scientific research. Vol.2, No.3, pp.124-131.

[21] Ranjan, G., Kumar, A. 2005. A natural disaster management system based on location aware distributed sensor networks, in proceeding of IEEE International Conference on Mobile Adhoc and Sensor Systems, 7 Nov. 2005 pp. - 182 
International Journal of Computer Applications Technology and Research

Volume 2- Issue 2, 141 - 146, 2013, ISSN: 2319-8656

[22] Saha, S., Matsumoto, M.2006. Performance Analysis of WSNDM (Wireless Sensor Network Protocol for
Disaster Management), International Conference on Communication Technology, ICCT '06.2006, pp 1 - 4 